\title{
The endurance shuttle walk: a new field test for the assessment of endurance capacity in chronic obstructive pulmonary disease
}

\author{
S M Revill, M D L Morgan, S J Singh, J Williams, A E Hardman
}

\begin{abstract}
Background-The purpose of this study was to develop an externally controlled, constant paced field walking test to assess endurance capacity in patients with chronic obstructive pulmonary disease (COPD). There were four objectives: (1) to develop a protocol; (2) to compare treadmill and shuttle walk tests of endurance capacity; (3) to examine the repeatability of the endurance shuttle walk test; and (4) to compare the sensitivity to pulmonary rehabilitation of endurance and incremental shuttle walk tests.

Methods-The test was designed to complement the incremental shuttle walk test (ISWT) using the same $10 \mathrm{~m}$ shuttle course and an audio signal to control

mean difference and limits of agreement (2SD) between tests 2 and 3 was +15 (42) s $(p>0.05)$. There was no significant change in performance on either test following the five week control period prior to rehabilitation. Following rehabilitation the ESWT duration increased by 160 (24)\% and the ISWT distance increased by 32 (11)\% (effect sizes 2.90 and 0.41 , respectively).

Conclusions-The ESWT was simple to perform, acceptable to all patients, and exhibited good repeatability after one practice walk. The test showed major improvement following rehabilitation and was more sensitive to change than the field test of maximal capacity.

(Thorax 1999;54:213-222)
\end{abstract} pace. The intensity of the field endurance test was related to a percentage of each patient's maximum field exercise performance assessed by the ISWT. A number of cassette tapes were prerecorded with a range of audio signal frequencies to dictate walking speeds between 1.80 and $6.00 \mathrm{~km} / \mathrm{h}$. In the first limb of the study 10 patients with COPD (mean (SD) forced expiratory volume in one second $\left(\mathrm{FEV}_{1}\right) \quad 1.0 \quad(0.36) \quad 1, \quad 35 \%$ predicted) performed three endurance shuttle walk tests (ESWTs) and three treadmill endurance tests. The walking speeds were calculated to elicit $75 \%, 85 \%$, and $95 \%$ of each patient's maximum ISWT performance for the field tests and measured peak oxygen consumption for the treadmill tests. In a separate group of patients the repeatability of the ESWT at an intensity of $85 \%$ of the ISWT performance was evaluated. Finally, the ESWT (at the $85 \%$ intensity) and the ISWT were performed at the start of a five week control period and at the start and end of a seven week pulmonary rehabilitation programme in 21 patients with COPD (mean $\mathrm{FEV}_{1} 0.80$ (0.18) 1).

Results-The mean (SE) times achieved during the ESWT were 13.1 (2.3), 10.2 (2.5), and $5.3(1.7) \mathrm{min}$ for the walks at $75 \%, 85 \%$, and $95 \%$ intensities, respectively. Patients tended to walk for longer on the treadmill than during the field tests at all intensities, but there were no significant differences between the end of test heart rates or Borg ratings of breathlessness or perceived exertion. Following one practice ESWT at the $85 \%$ intensity, the
Keywords: endurance shuttle walk test; chronic obstructive pulmonary disease; field exercise testing

Most activities of daily living represent exertion at submaximal exercise levels, so a measure of the ability to sustain a given submaximal exercise (endurance capacity) is an important component of the assessment of disability. In patients with chronic obstructive pulmonary disease (COPD) field walking tests are used as simple assessments of disability. The 12 and six minute field walk tests are self-paced ${ }^{12}$ and probably assess a combination of peak performance and endurance capacity. Their semistandardised nature contributes a degree of variability in the performance of the tests, ${ }^{34}$ and the imposition of a time limit constrains the validity of the tests as true measures of endurance capacity. A standardised field test of endurance capacity using constant walking speeds, external regulation of pace, and in which all patients experience a similar level of exercise intensity relative to their individual maximal capacity does not exist.

The development of a standardised, open ended field assessment of endurance capacity is desirable for the following reasons: (1) to overcome some of the difficulties associated with the existing timed walk tests-for example, variability in the patient selected walking speeds and in the operation of such tests between centres and studies; and (2) field tests are more flexible, accessible, and less costly than laboratory based tests.

The incremental shuttle walk test (ISWT) ${ }^{5}$ uses a $10 \mathrm{~m}$ course and the walking speed is externally controlled by signals from an audio cassette. Since this has been found to be a sat- 
Table 1 Mean (SD) patient data

\begin{tabular}{llll}
\hline & Group $A(n=10)$ & Group $B(n=11)$ & Group $C(n=21)$ \\
\hline M:F & $9: 1$ & $3: 8$ & $10: 11$ \\
FEV $_{1}(1)$ & $1.01(0.36)(35(4) \%$ pred $)$ & $0.79(0.21)(34(4) \%$ pred $)$ & $0.80(0.18)(35(8) \%$ pred $)$ \\
FVC $(1)_{\text {FEV }_{1} / \text { FVC (\%) }}$ & $2.92(0.55)(81(20) \%$ pred $)$ & $2.0(0.80)(68(14) \%$ pred $)$ & $2.06(0.90)(68(20) \%$ pred $)$ \\
Borg score (at rest) & $34(6)$ & $40(10)$ & $45(17)$ \\
Age (years) & $1.3(0.9)$ & $1.6(1.0)$ & $1.9(1.1)$ \\
Weight (kg) & $63.7(5.5)$ & $66.3(5.0)$ & $69(6.6)$ \\
\hline
\end{tabular}

$\mathrm{FEV}_{1}=$ forced expiratory volume in one second; FVC = forced vital capacity.

Group A performed the protocol development and comparison of treadmill and shuttle endurance walks, group B performed the repeatability limb, and group $\mathrm{C}$ performed the intervention limb.

isfactory method to standardise walking speed, the proposed test used a similar approachthat is, pre-recorded audio signals on a cassette tape and a $10 \mathrm{~m}$ shuttle course. The ISWT has been shown to produce a maximal response in patients with $\mathrm{COPD}^{6}$ so, in order to relate the walking speed of the field endurance shuttle walk test (ESWT) to a field measure of maximal exercise capacity, all patients performed an ISWT. Endurance capacity has been defined as the ability to sustain a high proportion of individual maximal oxygen uptake. ${ }^{7}$ We therefore investigated three endurance intensities which were related to $75 \%$, $85 \%$, and $95 \%$ of individual maximal capacity in order to establish a test of sufficient intensity to be practicable, yet not too lengthy for the patient and operator.

The aims of this study were: (1) to develop a shuttle walk test of endurance capacity; (2) to compare treadmill and shuttle walk tests of endurance capacity; (3) to examine the repeatability of the endurance shuttle walk test; and (4) to compare the sensitivity to pulmonary rehabilitation of endurance and incremental shuttle walk tests.

\section{Methods}

PATIENTS

Three groups of patients were recruited and they took part in the four limbs of the study as follows: group A $(n=10)$ participated in the development of the ESWT protocol and in the comparison of the treadmill and shuttle endurance tests; group B $(n=11)$ participated in the repeatability limb of the study; and group $\mathrm{C}$ ( $\mathrm{n}$ $=21$ ) was recruited to compare the sensitivity to pulmonary rehabilitation of endurance and incremental shuttle walk tests. Patients in

Table 2 Range of pre-recorded endurance walking speeds

\begin{tabular}{llll}
\hline Level & $\begin{array}{l}\text { Warm up speed } \\
(\mathrm{km} / \mathrm{h})\end{array}$ & $\begin{array}{l}\text { Endurance speed } \\
(\mathrm{km} / \mathrm{h})\end{array}$ & $\begin{array}{l}\text { Time/10 m } \\
\text { shuttle }(\mathrm{s})\end{array}$ \\
\hline 1 & 1.50 & 1.78 & 20.3 \\
2 & 1.50 & 2.09 & 17.3 \\
3 & 1.50 & 2.44 & 14.8 \\
4 & 1.50 & 2.72 & 13.3 \\
5 & 1.50 & 3.00 & 12.0 \\
6 & 2.40 & 3.27 & 11.0 \\
7 & 2.40 & 3.60 & 10.0 \\
8 & 2.40 & 3.79 & 9.5 \\
9 & 2.40 & 4.11 & 8.8 \\
10 & 2.40 & 4.36 & 8.3 \\
11 & 2.40 & 4.65 & 7.8 \\
12 & 2.40 & 4.97 & 7.3 \\
13 & 2.40 & 5.14 & 7.0 \\
14 & 2.40 & 5.54 & 6.5 \\
15 & 2.40 & 5.76 & 6.3 \\
16 & 2.40 & 6.00 & 6.0 \\
\hline
\end{tabular}

For each level the warm up speed was played for $100 \mathrm{~s}$ and the faster speed for the endurance test was played for $20 \mathrm{~min}$. group A were recruited from the outpatient respiratory clinics whilst patients in groups B and $\mathrm{C}$ were recruited from the waiting list for the pulmonary rehabilitation programme. Ten patients were common to both the repeatability and sensitivity limbs of the study.

COPD was diagnosed according to the British Thoracic Society guidelines ${ }^{8}$ and all patients were recruited on the basis of willingness to participate, evidence of moderate to severe airflow obstruction $\left(\mathrm{FEV}_{1}<60 \%\right.$ predicted), and self-reported exercise limitation due to breathlessness. Patients with cardiological, neurological, or locomotor disorders that limited exercise performance were excluded. All patients (with the exception of two) were on regular inhaled steroid treatment and all used inhaled bronchodilators. Three patients were receiving low dose oral steroids (prednisolone) and 12 patients were on diuretic therapy. Three patients recruited to the intervention limb (group C) were receiving domiciliary oxygen therapy for at least 15 hours per day and six patients were using oxygen for symptomatic relief only. There were no alterations to any medication during the study, although patients were instructed to use their inhaled bronchodilator within one hour of their study appointment time.

The study was approved by the district ethics committee and was fully explained before written informed consent was obtained from all participants. Height and weight were recorded at the start and weight was rechecked periodically during the study period. The $\mathrm{FEV}_{1}$ was measured at every visit using a wedge bellows spirometer (Model R, Vitalograph Ltd, Bucks, UK) according to the BTS/ARTP guidelines. ${ }^{9}$ Baseline breathlessness at rest was rated by Borg scales. ${ }^{10}$ Prediction values for static lung function variables were obtained from the European guidelines ${ }^{11}$ and prediction values for maximal exercise were obtained from the paper by Jones. ${ }^{12}$ The characteristics of the patients are shown in table 1 .

\section{MEASUREMENTS}

Development of the endurance shuttle walk test A series of signal frequencies were recorded onto a number of cassette tapes to provide an audio cue for constant walking over a range of speeds (table 2). Each tape started with a one minute calibration signal to ensure correct operation of the tape and cassette player followed by standardised instructions to the patient. A "warm up" slower pace, which lasted for approximately $100 \mathrm{~s}$, preceded the endurance speed to enable the patient to practise 


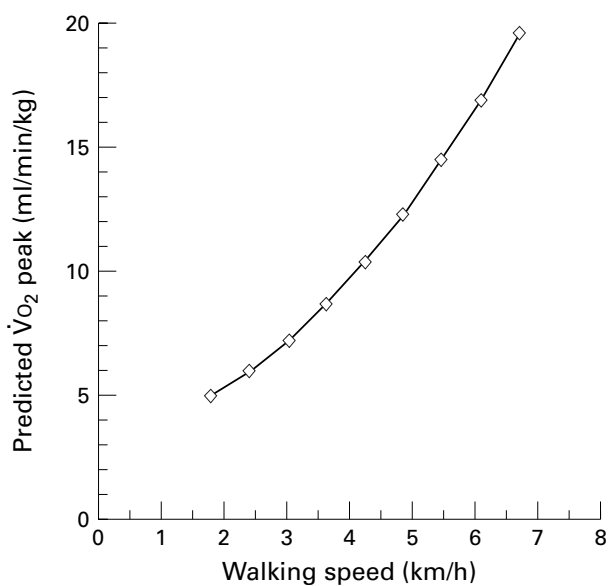

Figure 1 Incremental shuttle walking speeds and predicted oxygen consumption ( $\dot{V}_{\mathrm{O}_{2}}$ peak).

walking around the shuttle course. At the sound of a triple signal and a brief recorded message the signal frequency changed to the quicker endurance test speed which remained constant for 20 minutes.

The $10 \mathrm{~m}$ shuttle course was demarcated by cones on a quiet flat hospital corridor. Patients were instructed to walk along the course, turning around the cones at either end in time with the audio signals from the cassette player. The instructions were to continue walking until too tired or breathless to continue. For practical purposes we used a cut off time at 20 minutes, but patients were unaware of any time limit and were discouraged from estimating how long they had been walking.

To determine endurance exercise intensity, three intensities were investigated for the developmental limb of the study. In order to relate the walking speed of the field endurance tests to a percentage of individual maximum exercise capacity in the field an ISWT was performed. This enabled an approximate value for the peak oxygen consumption $\left(\dot{\mathrm{V}}_{2}\right.$ peak) to be predicted $^{6}$ and used as a reference point to determine the speed of the ESWT for each patient. The regression equation used to predict the $\dot{\mathrm{V}}_{2}$ peak from the ISWT distance was described by Singh et $a l^{6}$ and derived from a group of patients with a wide range of impairment $\left(\mathrm{FEV}_{1}\right.$ range $\left.0.5-3.11 \mathrm{l}\right)$. Values for $\dot{\mathrm{V}}_{2}$ equivalent to $75 \%, 85 \%$, and $95 \%$ of the predicted $\dot{\mathrm{V}}_{2}$ peak were calculated and the corresponding walking speeds interpolated from a graph which related shuttle walking speeds to predicted $\dot{\mathrm{V}}_{2}$ peak (fig 1). A cassette tape with signal frequencies closest to the derived speeds was selected from the bank of pre-recorded tapes. For example, patient 2 achieved $320 \mathrm{~m}$ in the ISWT which gave a predicted $\dot{\mathrm{VO}}_{2}$ peak of $12.2 \mathrm{ml} / \mathrm{min} / \mathrm{kg}$ (regression

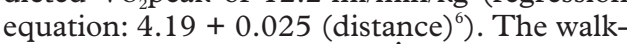
ing speeds which related to $\dot{\mathrm{V}}_{2}$ values of 9.1, 10.4 , and $11.6 \mathrm{ml} / \mathrm{min} / \mathrm{kg}(75 \%, 85 \%$, and $95 \%$ of the predicted $\dot{\mathrm{V}}_{2}$ peak) were read from the graph (fig 1) and ESWT tape levels 9, 10 and 12 were selected (table 2).

The heart rate was recorded during all field tests using a short range telemetry device (Sports tester PE3000, Polar Electro). The device consisted of a transmitter in a belt worn around the chest and a receiver worn around the wrist. Breathlessness and perceived exertion were rated from Borg scale ${ }^{10}$ immediately at the end of all the field exercise tests. The reasons for stopping exercise and the endurance exercise times were recorded.

\section{Treadmill tests of endurance capacity}

Cardiorespiratory measurements were made continuously during all of the treadmill tests using a computerised breath by breath exercise test system (OxyconBeta, Erich Jaeger). An ear oximeter (Biox 3700e, Ohmeda) provided simultaneous measurement of arterial oxygen saturation $\left(\mathrm{SaO}_{2}\right)$. Arterialised ear lobe capillary blood samples were taken at rest, at peak incremental treadmill exercise, and at the end of the $85 \%$ endurance treadmill test for the measurement of blood gas tensions and $\mathrm{pH}$. Additional ear lobe samples were taken at four minutes after exercise for the measurement of blood lactate concentration. Breathlessness and perceived exertion were rated immediately at the end of the treadmill exercise and the reasons for stopping exercise were also recorded.

In order to relate the intensity of the treadmill endurance tests to a percentage of the patient's maximum exercise capacity on a treadmill an incremental, symptom limited test was performed to establish individual values of $\dot{V}_{\mathrm{O}_{2}}$ peak. On a separate occasion a submaximal treadmill test was also performed in order to identify the relationship between oxygen uptake and treadmill walking speed for each patient. This submaximal test consisted of three different walking speeds of four minutes duration with a short rest period between each. The $\dot{\mathrm{V}}_{2}$ was measured during each stage and a graph of $\dot{\mathrm{V}}_{2}$ and the corresponding speed was plotted for each patient. The walking speeds which related to $75 \%, 85 \%$, and $95 \%$ of the measured $\dot{\mathrm{V}}_{2}$ peak for each patient were interpolated from their own individual graph. The three individualised walking speeds were used for the treadmill endurance tests.

The treadmill endurance tests were conducted in a manner similar to the field tests. The patients were instructed to walk for as long as possible. A slower "warm up" speed which lasted for $100 \mathrm{~s}$ was incorporated at the start of the tests and a 20 min time limit was imposed for the faster endurance speed. The patients remained unaware of the time limit; all clocks in the laboratory were turned out of sight and patients were not informed of how long they had been walking.

Repeatability of the endurance shuttle walk test The results from the developmental limb of the study showed that the ESWT at the 95\% intensity provoked a response similar to a maximum test for most of the patients, whilst $40 \%$ of the patients reached the 20 minute limit of the $75 \%$ ESWT. Thus, the most suitable endurance intensity for the field test, in terms of duration and patient acceptability, was the $85 \%$ intensity. This level of the ESWT was 
Table 3 Patients' responses at the end of the endurance shuttle walk tests

\begin{tabular}{lllll}
\hline & & & \multicolumn{2}{l}{ Borg ratings } \\
\cline { 4 - 5 } & Time & HR & HR \\
\cline { 4 - 5 } & (beats/min) & & $B S$ & $P E$ \\
\hline $75 \%$ ESWT & $13.1(2.3)$ & $117(5)$ & $3.9(0.4)$ & $13.5(0.6)$ \\
$85 \%$ ESWT & $10.2(2.5)$ & $121(6)$ & $4.5(0.6)$ & $13.6(0.9)$ \\
$95 \%$ ESWT & $5.3(1.7)^{\star}$ & $126(8) \dagger$ & $5.0(0.5)$ & $14.3(0.7)$ \\
\hline
\end{tabular}

$\mathrm{HR}=$ heart rate; $\mathrm{BS}=$ breathlessness; $\mathrm{PE}=$ perceived exertion. * Significantly different from $85 \%$ and $75 \%$ tests $(\mathrm{p}<0.05)$. †Significantly different from $75 \%$ test $(p<0.05)$

Values are mean (SE) measured at the end of each ESWT.

examined in the repeatability and sensitivity limbs of the study.

\section{Sensitivity of the endurance and incremental shuttle walk tests}

Patients in group C performed the endurance and the incremental shuttle walk tests at the start of a five week control period and at the start and end of a seven week outpatient pulmonary rehabilitation programme. The programme had educational and aerobic exercise components, and patients attended the hospital physiotherapy department for two hour classes twice weekly. The educational component consisted of interactive talks covering a variety of topics and a folder containing notes for the patient's future reference. The exercise training component consisted of a circuit of thoracic mobility and peripheral muscle strength training exercises in the physiotherapy gym (one hour per week) and a daily, home based walking programme. The walking training consisted of free walking-that is, not restricted to any circuit or equipment-for an individually prescribed time. The time was based on a treadmill endurance test performed during the initial pulmonary rehabilitation assessment. Patients kept a diary of walking time and were encouraged to increase the time throughout the seven weeks. Patients with oxygen desaturation $(<86 \%)$ during the initial assessment performed the exercise training with portable oxygen.

STUDY DESIGN

Group A completed the developmental limb of the study which involved 10 separate visits. Following the incremental tests to establish individual maximal exercise capacity on the treadmill and in the "field", three treadmill endurance tests and the three ESWTs (relating to $75 \%, 85 \%$, and $95 \%$ of maximal exercise capacity) were presented in randomised order on separate occasions. For the repeatability limb of the study patients in group B made three visits to the hospital over a period of 15 days. At the first visit the ISWT was performed and after a rest period of at least 40 minutes an ESWT at $85 \%$ intensity was performed. The $85 \%$ ESWT was repeated during visits 2 and 3 . For the sensitivity limb of the study patients in group C made four separate visits. Following a pre-study familiarisation visit at which practice shuttle tests were performed, the ESWT at the $85 \%$ intensity and an ISWT were measured at the start of a control period (no change in treatment) and at the start and the end of the pulmonary rehabilitation programme.

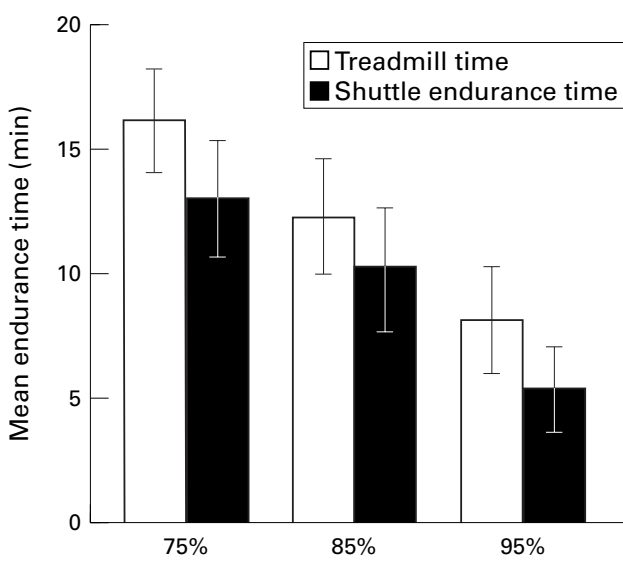

Figure 2 Mean (SE) shuttle and treadmill endurance times.

STATISTICAL ANALYSIS

Summary data are expressed as mean (SD) unless otherwise stated, and a 5\% level of significance was adopted throughout. Relationships between variables were examined using the Pearson product moment correlation coefficient $(r)$ and the method of least squares was used for any regression analysis. The Student's $t$ test was used to examine the difference between two correlated means and analysis of variance (ANOVA) used for multiple comparisons. The Scheffe post hoc test was used to determine where significant differences occurred. The results of the Borg ratings were subject to appropriate non-parametric analysis.

\section{Results}

DEVELOPMENT OF THE ESWT

All patients in group A completed the developmental limb with an average time between visits of 10 (4) days. The endurance capacity was defined as the duration of walking at the set endurance speed. The mean walking speeds for the three ESWTs were 3.8 (0.72), 4.1 (0.71), and $4.4(0.71) \mathrm{km} / \mathrm{h}$ for the $75 \%, 85 \%$, and $95 \%$ tests, respectively. There was a progressive decrease in the endurance times as the intensity increased (table 3). One patient completed 20 minutes in all three ESWTs. As a precaution this patient repeated the ISWT to ensure a peak performance had been achieved but the result was identical. There was a trend for the Borg ratings for breathlessness to increase as the endurance intensity increased, but there were no significant differences between ratings. The same was true for the ratings of perceived exertion (table 3 ).

There was no relationship between the $\mathrm{FEV}_{1}$ and the endurance times. There was a moderate and statistically significant relationship between the $75 \%$ and the $85 \%$ endurance times $(r=0.83, \mathrm{p}<0.05)$, but this was influenced by the number of patients attaining the externally imposed 20 minute time limit in both tests.

The mean distance walked during the ISWT was $313(96) \mathrm{m}$ with a peak heart rate of 123 (20) beats/min. The mean duration of the ISWT was $345(156) \mathrm{s}$. The mean Borg ratings at the end of the ISWT were 4.9 (1.9) for 
Table 4 Mean (SE) responses to treadmill tests to determine $\dot{V}_{2}$ peak and endurance capacity

\begin{tabular}{|c|c|c|c|c|c|c|c|c|}
\hline Test & $\begin{array}{l}\dot{\text { Vo }} \text { opeak } \\
(\mathrm{mllmin} / \mathrm{kg})\end{array}$ & $\dot{V}_{E}(l / m i n)$ & $\begin{array}{l}\text { HR } \\
\text { (beats/min) }\end{array}$ & $V t(m l)$ & $\begin{array}{l}f R \\
\text { (breaths/min) }\end{array}$ & $R E R$ & $B S$ & $P E$ \\
\hline \multicolumn{9}{|l|}{ Endurance } \\
\hline $75 \%$ & $12.1(0.5)$ & $33.4(3.1)$ & $112(7)$ & $1287(104)$ & $26.6(2.1)$ & $0.87(0.01)$ & $4.1(0.7)$ & $13.2(0.9)$ \\
\hline $85 \%$ & $13.4(0.9)$ & $37.2(4.2)$ & $114(6)$ & $1394(126)$ & $26.8(1.7)$ & $0.90(0.02)$ & $4.5(0.5)$ & $14.0(0.8)$ \\
\hline $95 \%$ & $15.1(1.0)^{\star}$ & $40.5(3.4)^{\star}$ & $122(7) \dagger$ & $1444(103)^{\star}$ & $28.5(2.1)$ & $0.92(0.02)$ & $5.8(0.7)^{\star}$ & $15.3(0.7)^{\star}$ \\
\hline Incremental & $15.5(1.2)$ & $42.1(4.0)$ & $120(6)$ & $1614(158)$ & $28.2(1.8)$ & $0.91(0.02)$ & $5.5(0.5)$ & $15.4(0.6)$ \\
\hline
\end{tabular}

$\dot{\mathrm{V}}_{2}$ peak = oxygen consumption; $\dot{\mathrm{V}}_{\mathrm{E}}=$ ventilation; $\mathrm{HR}=$ heart rate; $\mathrm{Vt}=$ tidal volume; $\mathrm{fR}=$ respiratory rate; $\mathrm{RER}=$ respiratory exchange ratio; $\mathrm{BS}=$ breathlessness; $\mathrm{PE}=$ perceived exertion.

Measurements were from the final minute of each endurance test and the symptom limited incremental treadmill test.

^Significantly different from $75 \%$.

†Significantly different from $75 \%$ and $85 \%(\mathrm{p}<0.05)$

Incremental $\dot{\mathrm{V}}_{2}$ peak $=1.09(0.08) \mathrm{l} / \mathrm{min}$

breathlessness and 13.3 (1.9) for perceived exertion. Breathlessness was identified as the main cause of exercise termination during the ISWT.

TREADMILL ENDURANCE TESTS AND COMPARISON WITH SHUTTLE TESTS

The mean walking speeds for the three treadmill endurance tests were $3.4(1.0), 4.0$ (1.1), and $4.7(1.2) \mathrm{km} / \mathrm{h}$ for the $75 \%, 85 \%$, and $95 \%$ tests, respectively. The mean (SE) times were $16.2(2.1), 12.3$ (2.3), and 8.1 (2.2) $\mathrm{min}$ for the $75 \%, 85 \%$, and $95 \%$ tests, respectively (fig 2). Although patients tended to walk for longer on the treadmill than around the shuttle course, there were no significant differences between times. There were wide differences in some patients, however, and subsequently there were no significant relationships between the treadmill and field test times except for the $75 \%$ tests $(r=0.68, \mathrm{p}<0.05)$. This relationship was, however, influenced by the number of patients who attained the externally imposed 20 minute limit. There were no significant differences between the heart rate at the end of the test and the Borg ratings at comparable field and treadmill endurance levels.

The mean values of $\mathrm{SaO}_{2}$ at the end of the treadmill endurance exercise were 93.0 $(2.1) \%, 92.4(3.1) \%$, and $91.7(2.4) \%$ for the $75 \%, 85 \%$, and $95 \%$ tests, respectively. The end of test values for $\dot{\mathrm{V}}_{2}$, ventilation $(\dot{\mathrm{V}} \mathrm{E})$, and heart rate showed a progressive increase at each level of endurance intensity (table 4). There was wide variability in the pattern of the $\dot{\mathrm{V}}_{2}$ responses at the same endurance intensities. Four patients attained a steady state in their $\dot{\mathrm{V}} \mathrm{O}_{2}$ during the $75 \%$ test and two patients had a rapid increase to reach a peak response at this level (final $\dot{\mathrm{V}}_{2} \geqslant \dot{\mathrm{V}}_{2}$ peak). The remaining patients had a gradual increase in $\dot{\mathrm{V}}_{2}$. Five patients attained a peak response during the 95\% test and two patients achieved a steady state. The mean percentages of individual

Table 5 Mean (SE) blood gas tensions and blood lactate concentration following incremental and $85 \%$ endurance treadmill tests

\begin{tabular}{|c|c|c|c|c|}
\hline & $\mathrm{PaO}_{2}(\mathrm{kPa})$ & $\mathrm{PaCO}_{2}(\mathrm{kPa})$ & $p H$ & Lactate (mmoll/) \\
\hline \multicolumn{5}{|c|}{ Incremental } \\
\hline Rest & $9.88(0.32)$ & $4.84(0.06)$ & $7.423(0.007)$ & $0.39(0.14)$ \\
\hline Peak & $8.03(0.24)$ & $4.87(0.18)$ & $7.391(0.012)$ & $1.22(0.52)$ \\
\hline \multicolumn{5}{|l|}{$85 \%$} \\
\hline Rest & $10.42(0.44)$ & $4.74(0.10)$ & $7.426(0.007)$ & $0.41(0.12)$ \\
\hline Peak & $8.50(0.42)$ & $4.92(0.13)$ & $7.386(0.009)$ & $1.34(0.56)$ \\
\hline
\end{tabular}

$\mathrm{PaO}_{2}, \mathrm{PaCO}_{2}=$ arterial oxygen and carbon dioxide tensions

Values are for rest and peak exercise, except for blood lactate concentration which was measured four minutes after exercise.
$\dot{\mathrm{V}}_{2}$ peak achieved during the final minute of each endurance test were $81(16) \%, 87(13) \%$, and $97(11) \%$ for the $75 \%, 85 \%$, and $95 \%$ tests, respectively.

The main reasons for terminating exercise during the $75 \%$ and $85 \%$ tests were attainment of the 20 minute limit or leg fatigue and general fatigue for both the ESWT and the treadmill endurance tests. Breathlessness was cited more often during the $95 \%$ endurance tests.

For comparison purposes the results of the symptom limited incremental treadmill test are also shown in table 4. The $\mathrm{V}_{2}$ peak of the group was lower than the predicted normal value $(55 \%)$ and there was a moderate heart rate reserve $(73 \%$ predicted). There was a degree of oxygen desaturation with the $\mathrm{SaO}_{2}$ falling from $95.4(1.5) \%$ at rest to $91.5(2.4) \%$ during the incremental maximum test. The mean fall was $3.9(2.6) \%$. There was also a fall in the $\mathrm{PaO}_{2}$, but little change in the $\mathrm{PaCO}_{2}$. The blood gas analysis and lactate concentrations are shown in table 5 .

There was no relationship between baseline $\mathrm{FEV}_{1}$ measurements and the treadmill times. A significant relationship was found between the fall $(\Delta)$ in $\mathrm{SaO}_{2}$ and the $85 \%$ endurance time $(r$ $=0.72, \mathrm{p}<0.025)$. The relationship between the fall in $\mathrm{SaO}_{2}$ and the $75 \%$ treadmill time was of borderline significance $(r=0.62, \mathrm{p}=0.06)$. There were significant relationships between the end of test field and treadmill heart rates $r$ $=0.90,0.76$, and 0.73 , respectively, for the $95 \%, 85 \%$, and $75 \%$ tests, $\mathrm{p}<0.025$; fig 3 ).

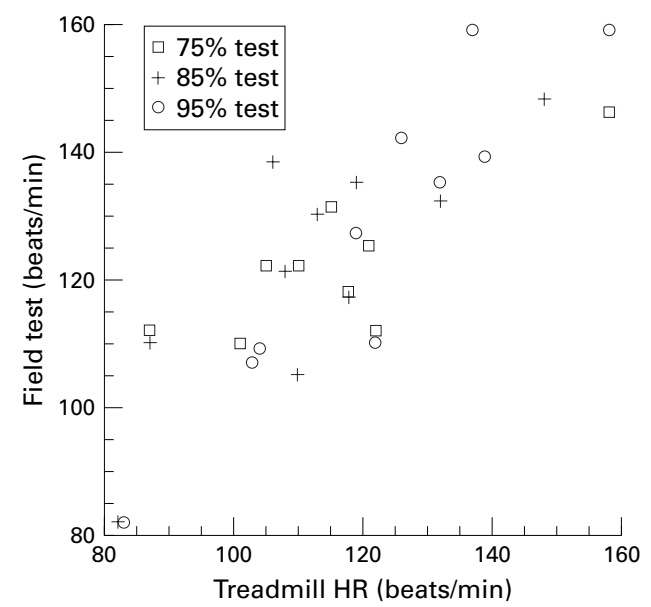

Figure 3 Individual heart rates (HR) at the end of the shuttle and treadmill endurance tests. 
Table 6 Repeatability of the 85\% endurance shuttle walk test $(n=11)$

\begin{tabular}{|c|c|c|c|c|c|}
\hline \multirow[b]{2}{*}{ Visit } & \multirow[b]{2}{*}{ Time $(s) t$} & \multirow[b]{2}{*}{ Distance $(m) \dagger$} & \multirow[b]{2}{*}{$H R$ (beats/min) $\dagger$} & \multicolumn{2}{|c|}{ Borg ratings } \\
\hline & & & & $B S t$ & PE† \\
\hline 1 & $251(120)^{\star}$ & $214(101)^{\star}$ & $108(17)$ & $4.6(2.0)$ & $12.7(1.9)$ \\
\hline 2 & $310(171)$ & $262(137)$ & $107(16)$ & $4.4(1.6)$ & $12.6(2.1)$ \\
\hline \multirow[t]{2}{*}{3} & $325(158)$ & $274(130)$ & $103(16)$ & $4.5(1.7)$ & $12.9(2.7)$ \\
\hline & & \multicolumn{2}{|c|}{ Time $(s) \neq$} & \multicolumn{2}{|c|}{ Distance $(m) \neq$} \\
\hline \multicolumn{2}{|l|}{ Test 2 vs 1} & \multicolumn{2}{|c|}{59.5 (22.4) $(95 \%$ CI 9.7 to 109.2$)$} & \multirow{2}{*}{\multicolumn{2}{|c|}{47.5 (18.4) (95\% CI 6.6 to 18.4$)$}} \\
\hline \multirow{2}{*}{\multicolumn{2}{|c|}{$\begin{array}{l}\text { Test } 3 \text { vs } 1 \\
\text { Test } 3 \text { vs } 2\end{array}$}} & \multicolumn{2}{|c|}{74.3 (18.8) (95\% CI 32.5 to 116.1$)$} & & \\
\hline & & $14.8(6.3)(95 \%$ & CI 0.6 to 28.9 ) & \multicolumn{2}{|c|}{$\begin{array}{l}60.3 \text { (16.2) }(95 \% \text { CI } 24.1 \text { to } 96.5 \\
12.8 \text { (4.9) }(95 \% \text { CI } 1.9 \text { to } 23.6)\end{array}$} \\
\hline
\end{tabular}

$\mathrm{HR}=$ heart rate $; \mathrm{BS}=$ breathlessness $; \mathrm{PE}=$ perceived exertion.

${ }^{\star}$ Signficantly different from tests 2 and $3(\mathrm{p}<0.05)$.

†Mean (SD) values for three $85 \%$ ESWTs performed over a 15 day period.

$\ddagger$ Mean (SE) and $95 \%$ confidence intervals for the differences between repeat tests.

Table 7 Endurance (ESWT) and incremental shuttle walk tests (ISWT) before and after rehabilitation $(n=21)$

\begin{tabular}{|c|c|c|c|c|c|}
\hline & Time (s) & Distance (m) & $\begin{array}{l}H R \\
\text { (beats/min) }\end{array}$ & $B S$ & $P E$ \\
\hline \multicolumn{6}{|l|}{ ESWT } \\
\hline Control & $265(34)$ & $228(30)$ & $113(4)$ & $4.3(0.4)$ & $12.5(0.5)$ \\
\hline Start rehabilitation & $\begin{array}{l}270(31) \\
5(-20 \text { to } 30)\end{array}$ & $\begin{array}{l}237(31) \\
9(-12 \text { to } 30)\end{array}$ & $112(4)$ & $4.2(0.3)$ & $12.1(0.3)$ \\
\hline End rehabilitation & $\begin{array}{l}674(86)^{\star} \\
404(264 \text { to } 544)\end{array}$ & $\begin{array}{l}571(78)^{\star} \\
334(211 \text { to } 450)\end{array}$ & $116(4)$ & $3.7(0.3)^{\star}$ & $11.6(0.4)^{\star}$ \\
\hline \multicolumn{6}{|l|}{ ISWT } \\
\hline Control & - & $175(17)$ & $113(4)$ & $5.1(0.4)$ & $12.7(0.3)$ \\
\hline Start rehabilitation & - & $\begin{array}{l}178(20) \\
3(-15 \text { to } 22)\end{array}$ & $106(4)$ & $4.6(0.4)$ & $13.9(0.5)$ \\
\hline End rehabilitation & - & $\begin{array}{l}215(20)^{\star} \\
36(11 \text { to } 61)\end{array}$ & $111(4)$ & $3.9(0.3)^{\star}$ & $12.1(0.4)^{\star}$ \\
\hline
\end{tabular}

*Significantly different from start $(\mathrm{p}<0.05)$.

Mean (SE) time, distance walked, heart rate (HR) at the termination of exercise, and Borg breathlessness (BS) and perceived exertion (PE) at the start of the control period and at the start and end of rehabilitation. The mean differences (95\% confidence intervals) between the start and end of the control period (visit 3-2), and the start and end of the rehabilitation programme (visit 4-3) are also shown.
SENSITIVITY TO REHABILITATION OF THE ESWT AND ISWT

Four patients had a fall in oxygen saturation to $<86 \%$ during the initial practice ISWT and received ambulatory oxygen for all subsequent tests. In addition, these patients also received ambulatory oxygen during the exercise training component of the rehabilitation programme. The mean resting $\mathrm{SaO}_{2}$ for the whole group was $94.5(1.5) \%$. At the end of the initial familiarisation ISWT the $\mathrm{SaO}_{2}$ was 90.8 (3.7)\% and the mean fall was $3.7(3.0) \%$.

The mean duration of the control period was 5 (1.2) weeks and the mean endurance walking speed at $85 \%$ intensity was $3.02(0.75) \mathrm{km} / \mathrm{h}$. There was no significant change in performance on the ESWT at the start and end of the control period, the mean (SE) difference in time spent walking being +5 (11.9) $\mathrm{s}$ and the difference in distance walked was +9.3 (10.3) $\mathrm{m}(\mathrm{p}>0.05)$. Similarly, there was no significant change in performance on the ISWT (mean difference $+3.0(9.0) \mathrm{m}$ ).

All the patients with the exception of patient 10 had an improvement in the ESWT at the end of the rehabilitation course. Prior to rehabilitation the maximum time achieved by any single patient during the ESWT was $709 \mathrm{~s}$ $(11 \mathrm{~min} 49 \mathrm{~s})$. Following rehabilitation three patients completed $1200 \mathrm{~s} \mathrm{(20} \mathrm{min)} \mathrm{and} \mathrm{four}$ patients achieved $>17 \mathrm{~min}$. The response to the ISWT was more variable with 10 patients having an improvement of $\geqslant 30 \mathrm{~m}$ and in 11 patients the ISWT distance was within $29 \mathrm{~m}$ of their pre-rehabilitation test. There were significant improvements in both the ESWT and ISWT for the whole group. The mean percentage improvement in endurance capacity was $160(110) \%$ and for the ISWT was $32(50) \%$. There was a large effect size for the ESWT (2.9) and a moderate effect size for the ISWT (0.41). A summary of the walking test results for both the ESWT and ISWT are shown in table 7 and fig 5 .

Only one patient did not show an improvement in the ESWT following rehabilitation (patient 10). A slight decrease in the endurance capacity was measured (436 s at the start of rehabilitation and $399 \mathrm{~s}$ at the end). To eliminate the possibility of a spurious result the post-rehabilitation ESWT was repeated at a separate visit. The results were within four seconds (399 s and $395 \mathrm{~s}$ ). Additionally, there was minimal improvement in the ISWT. This patient died suddenly three weeks after completing the course.

There were significant improvements in the Borg ratings for dyspnoea and perceived exertion following rehabilitation for both the ESWT and the ISWT (table 7). The reasons for the termination of endurance exercise did not change appreciably during the control period. Following rehabilitation, fewer patients identified dyspnoea ( 8 vs $13, \mathrm{p}=0.07$ ) and more patients identified tiredness as the main symptom following the ESWT (5 vs 1, $\mathrm{p}<0.05)$. The frequencies of the reasons for exercise termination are shown in fig 6 . The main reason for termination of the ISWT was
Figure 4 Differences in $85 \%$ shuttle endurance time between tests 2 and $3(n=11)$. 

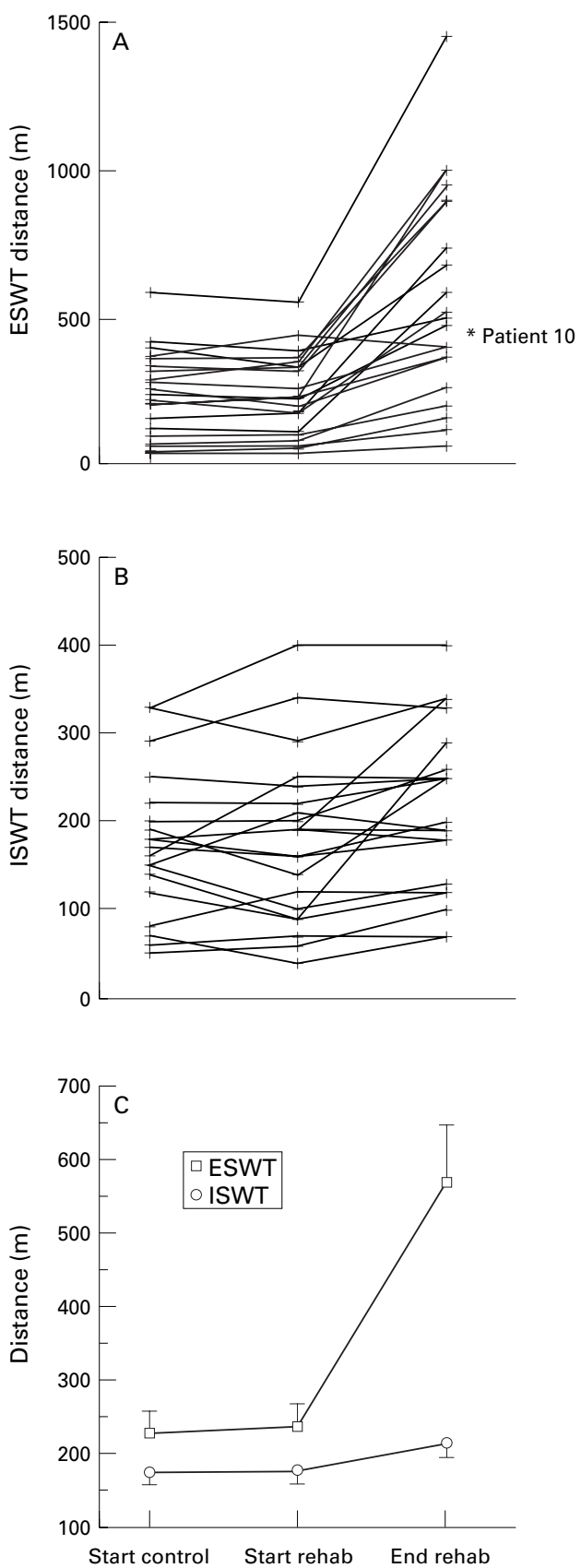

Figure 5 Individual $(A)$ endurance and $(B)$ incremental shuttle walking distances and (C) mean (SE) distances for both tests at the start of the control period and at the start and end of rehabilitation.

dyspnoea and this remained unchanged following rehabilitation.

There were no significant changes in the peak heart rate response following both the ESWT and the ISWT during the control period and following rehabilitation. The mean (SE) heart rate responses at equivalent endurance times before and after rehabilitation were 111 (4.0) and 111 (3.9), respectively.

\section{Discussion}

We have developed an endurance field test with an externally controlled, constant walking speed to achieve standardisation of exercise intensity and to give patients the opportunity to sustain exertion until limited by symptoms.
The test showed good repeatability after one practice walk and was sensitive to change following pulmonary rehabilitation. The adaptation of the ISWT format has shown that standardisation of constant walking speed is achievable, and the intensity of exercise may be determined individually from the peak field performance measured during the ISWT. The test was acceptable to all the patients in the study, it was simple to perform, required minimal inexpensive equipment, and might be used in any quiet enclosed space of $>10 \mathrm{~m}$.

DEVELOPMENTAL LIMB

At each relative intensity the group achieved longer endurance times on the treadmill with slightly lower heart rates than with the equivalent ESWT, which suggests that treadmill walking provoked less physiological stress. The differences in response to the test modalities probably reflect a number of fundamental differences between treadmill and free walking. There is lower energy expenditure on the treadmill as a result of the belt assisting the backward movement of the foot and there are differences in shoe to ground friction and step impact. ${ }^{13}$ There is likely to be higher energy expenditure during the shuttle walk as a result of turning the corners of the circuit compared with walking in a straight line on the treadmill. However, the heart rate responses provoked by the ESWT were similar to those achieved on the treadmill, with significant relationships between comparable levels of endurance. This finding supports the use of the ESWT to provoke a similar cardiac response to that produced at a comparable intensity on the treadmill.

The patients were given no indication of how long they had been walking and, to avoid setting targets, were not informed of the 20 minute limit. In this sense the ESWT was open ended, in contrast to the six or 12 minute walk tests which are time limited and self-paced. Previous studies have shown that knowledge of the imposed time limit of a test influences pacing and the distance covered. ${ }^{3}$ Such findings emphasise the non-standardised nature of the timed walk tests which rely on individual judgement of pace and a self-imposed work rate. Whilst it may be argued that self-pacing is a valuable skill to be learnt by severely limited patients, ${ }^{14}$ the imposition of a stated time limit constrains the validity of the tests as true measures of endurance capacity. In addition, the adoption of a habitual walking pace will impose a natural limit to any increase following an intervention and may mask the full beneficial effect of any treatment. ${ }^{15}$ Any variability in work rate was avoided with the ESWT where the speed was externally imposed, so the test is likely to be a more rigorous measure of longitudinal change than self-paced tests.

A short endurance test is desirable in terms of practical application and for the wider margin for change following an intervention. However, four patients completed three minutes or less during the $95 \%$ test whilst four patients completed the full 20 minutes of the $75 \%$ ESWT. The results from this study suggest that 


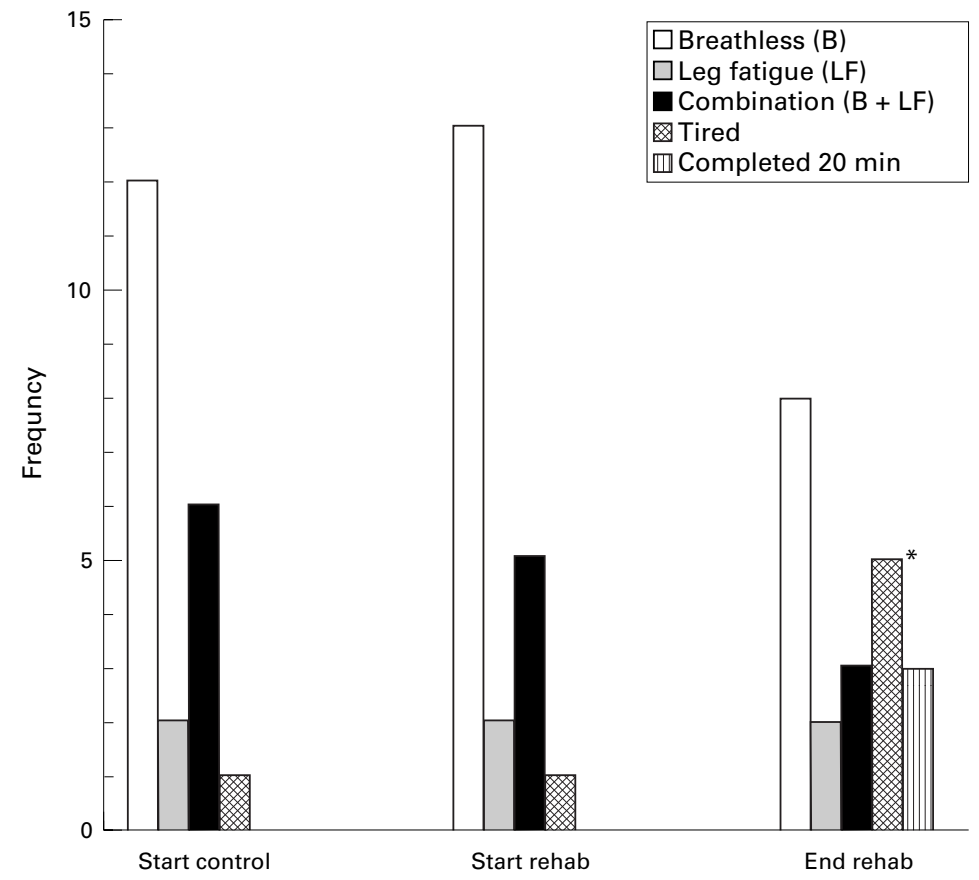

Figure 6 Frequency of occurrence of symptoms at the termination of shuttle endurance exercise. $\left({ }^{*} p<0.05\right)$.
$11 /$ min of oxygen in healthy individuals. ${ }^{13}$ This level of oxygen consumption is close to the $\dot{\mathrm{V}}_{2}$ peak measured in the patients in this study. The ability of the patients to exercise at high intensities relative to $\dot{\mathrm{V}}_{2}$ peak supports the findings of others ${ }^{17} 18$ where high intensity training and testing have been used in rehabilitation programmes. The provocation of high proportions of individual maximum capacity by relatively low absolute work loads has implications for exercise prescription and training intensity in this patient group.

\section{REPEATABILITY}

A positive improvement in $85 \%$ ESWT was measured with each successive performance of the test, and suggested a diminishing learning effect in the test performance. Although the first test was performed on the same day as the ISWT, there was a rest period of at least 40 minutes between tests so the development of undue tiredness was unlikely. The third test improved the ESWT duration by an average of only $15 \mathrm{~s}$ compared with an increase of $60 \mathrm{~s}$ following the first test. Thus, reasonably good test repeatability was established following one practice walk, though imprecision in the limits of repeatability may be present as a result of the small sample size. However, the mean (SE) difference of +15 (6) s (distance $=13(5) \mathrm{m}$ ) compares favourably with a repeatability of +41 (18) $\mathrm{m}$ for the 12 minute walk test, ${ }^{1}$ and +2 (10) $\mathrm{m}$ for the ISWT. ${ }^{5}$ In routine clinical use the ISWT, the practice ESWT, and actual ESWT may be required on the same day, in which case rest periods of at least 40 minutes should be allowed between tests. The smaller mean difference between the tests carried out at the start and end of the five week control period of the intervention limb suggests that the learning effect diminished with time, whilst greater individual variability is suggested from the wider confidence interval (50 s).

\section{SENSITIVITY TO REHABILITATION}

The magnitude of improvement in the ESWT was far greater than the improvement in the ISWT (effect size 2.90 vs 0.41 ). With the exception of one patient, the improvements in the ESWT were large (minimum improvement $30 \%$ ) and consistent. These findings support the contention that tests of endurance capacity exhibit greater sensitivity to change than tests of maximum capacity following rehabilitation in patients with COPD.

The relatively greater improvement in the ESWT emphasised that endurance capacity is likely to be more amenable to exercise training in this patient group where the pathophysiology of COPD may limit improvements in the maximum capacity. Following rehabilitation, three patients attained the 20 minute limit of the ESWT and four patients achieved more than 17 minutes. Only three of these seven patients had significant improvements in the ISWT which suggests that possible improvements in maximum aerobic capacity cannot wholly explain the changes in endurance exercise for some patients. The variable changes in the ISWT contrast with the large and consist- 
ent improvements in the ESWT and emphasise the need to examine endurance capacity when pulmonary rehabilitation is undertaken.

The improvements in the $85 \%$ ESWT indicated that the changes in endurance capacity were not confined to the training intensity (all the patients confirmed that the ESWT speed was faster than they would normally walk). A study which closely examined training intensity in patients with severe COPD found that most of the patients were unable to achieve a target training intensity of $80 \%$ maximum work rate on a cycle ergometer. ${ }^{19}$ However, there were significant improvements in exercise tolerance following the rehabilitation and evidence of physiological adaptation to training. The current study and others ${ }^{19}$ suggest that useful improvements can be achieved with moderate training intensities; however, in severely limited patients with reduced $\dot{\mathrm{V}}_{2}$ peak, low and moderate absolute work rates are likely to engage a high proportion of the $\dot{\mathrm{V}}_{2}$ peak.

The purpose of predicting a value for the $\dot{\mathrm{V}}_{2}$ peak from the ISWT is to provide a reference for the maximum performance achieved during field exercise. Equally, the individual maximum walking speed might offer a suitable reference from which the intensity of endurance exercise can be derived. In the intervention limb of the study the mean ESWT walking speed for the group was 3.02 $(0.75) \mathrm{km} / \mathrm{h}$ which equated to 83 (5) \% of the maximum speed achieved during the initial ISWT. Whether the post-training assessments should be conducted at pre-training intensities is an important question and forms a natural adjunct of further research to the current study. In the case of patients achieving the 20 minute limit, a simple reassessment at the next endurance walking speed on the ESWT tape may form a practical solution which warrants further investigation.

Most patients improved their walking duration with similar or reduced ratings of dyspnoea and perceived exertion. There was no change in the heart rate response which suggests there was no cardiac training effect. However, the improvements in endurance capacity and lowered sense of perceived exertion would support a hypothesis of enhanced mechanical efficiency. Improved skill of performance and coordination will lead to improved efficiency and have a sparing effect on $\dot{\mathrm{VO}}_{2}$. Such a mechanism would suggest more efficient use of existing capacity and may partly explain why many of the patients had an increased endurance capacity without concurrent improvements in maximal capacity.

Only one patient did not improve following the rehabilitation (patient 10). The patient died within a few weeks of completing the course and details of the sudden death suggested it was of cardiac origin. The lack of improvement suggested that other covert clinical problems may have acted to negate any therapeutic effects from the rehabilitation.

LIMITATIONS OF THE STUDY

The sample size for the repeatability limb of the study was small and this may have resulted in some imprecision in the limits of agreement. However, the repeatability following one practice walk was at least as good as other field measures of exercise. The stability of the ESWT over shorter and longer periods-for example, within a day and over 6-8 weeks-requires further investigation in addition to the establishment of the minimum clinical important difference. The ESWT is more complex to administer at the initial testing stage, requiring the performance of the ISWT to enable the endurance speed to be calculated. In addition, the derivation of the speed might be simplified by using a percentage of the maximum ISWT speed achieved. However, we would always recommend the measurement of both maximal and endurance capacity to assess disability and the effects of rehabilitation in COPD. During the intervention limb we used each patient as their own control rather than having a separate parallel control group. Furthermore, we did not use a sham rehabilitation programme to control for placebo effects. Whilst there is no consensus for the type, frequency, and intensity of exercise training in this patient group, many of the published studies report a variety of approaches. We feel the benefits of the exercise training component of pulmonary rehabilitation have already been firmly established following a review of 14 randomised controlled trials and the publication of evidence based guidelines. ${ }^{20}$

In summary, the ESWT was sensitive to pulmonary rehabilitation, the test amplified the training induced changes, and large improvements were seen in most patients. The changes suggest that the rehabilitation acted to optimise the use of residual functional capacity, whilst its impact on maximal capacity was more variable where improvement may be limited by the extent of pulmonary impairment. The developmental study highlighted the differences between treadmill walking and free walking around the shuttle circuit. Although the heart rate responses were similar, patients walked for longer on the treadmill, suggesting lower physiological stress. The ESWT benefits from standardised walking speeds and allows the same exercise challenge to be repeated longitudinally. It is simple to perform, requiring only minimal equipment, and has good repeatability over the short and medium term. This study has demonstrated large improvements in the ESWT following pulmonary rehabilitation, and endorses the need to examine endurance capacity where this intervention is used in the clinical management of patients with COPD.

The endurance shuttle walk test is available on cassette tape The tapes and instruction leaflet may be obtained from Dr S M Revill, Department of Respiratory Medicine, Glenfield HospiRevill, Department of Respirat
tal, Leicester LE3 9QP, UK.

1 McGavin CR, Gupta SP, McHardy GJR. Twelve-minute walking test for assessing disability in chronic bronchitis. BMF 1976;1:822-3

2 Butland RJA, Pang J, Gross ER, et al. Two-, six-, and 12 -minute walking tests in respiratory disease. BMF 1982; 284:1607-8.

3 Guyatt GH, Pugsley SO, Sullivan MJ, et al. Effect of encouragement on walking test performance. Thorax 1984; 39:818-22.

4 Knox AJ, Morrison JFJ, Muers MF. Reproducibility of walking test results in chronic obstructive airways disease. Thorax 1988;43:388-92. 
5 Singh SJ, MDL Morgan, Scott S, et al. Development of a shuttle walking test of disability in patients with chronic 6 ain SJ, Moruction. Tho

6 Singh SJ, Morgan MDL, Hardman AE, et al. Comparison of oxygen uptake during a conventional treadmill test and the shuttle walking test in chronic airflow limitation. Eur Respir f 1994; 7:2016-20.

7 Hardman AE. Exercise training. In: Morgan MDL, Singh SJ, eds. Practical pulmonary rehabilitation. London: Chapman and Hall, 1997: 63-80

8 British Thoracic Society. BTS guidelines for the management of chronic obstructive pulmonary disease. Thorax 1997;52(Suppl 5):S1-28.

9 BTS/ARTP. Guidelines for the measurement of respiratory function. Respir Med 1994;88:165-94.

10 Borg GAV. Psychophysical basis of perceived exertion. Med Sci Sports Exerc 1982;14:377-81.

11 Quanjer PH, ed. Standardization of lung function tests update. Report of Working Party for the European Community for Steel and Coal. Eur Respir $\mathcal{f} 1993$; 6(Suppl): 16

12 Jones NL. Clinical exercise testing. 3rd edn. Philadephia: WB Saunders, 1987.

13 Åstrand PO, Rodahl K. Textbook of work physiology: physiological bases of exercise. 3rd edn. New York: McGraw Hill, 1986.
14 Steele B. Timed walking tests of exercise capacity in chronic cardiopulmonary illness. F Cardiopulm Rehab 1996;16:2533

15 Swinburn CR, Wakefield JM, Jones PW. Performance, ventilation, and oxygen consumption in three different types of exercise test in patients with chronic obstructive lung disease. Thorax 1985;40:581-6.

16 Matthews JI, Bush BA, Ewald FW. Exercise responses during incremental and high intensity and low intensity steady state exercise in patients with obstructive lung disease and normal control subjects. Chest 1989;96:11-7.

17 Ries AL, Archibald CJ. Endurance exercise training at maximal targets in patients with chronic obstructive pulmonary disease. ff Cardiopulm Rehab 1987;7:594601.

18 Punzal PA, Ries AL, Kaplan RM, et al. Maximum intensity exercise training in patients with chronic obstructive pulmonary disease. Chest 1991;100:618-23.

19 Maltais F, LeBlanc P, Jobin J, et al. Intensity of training and physiologic adaptation in patients with chronic obstructive pulmonary disease. Am f Respir Crit Care Med 1997;155: 555-61.

20 Pulmonary Rehabilitation Joint ACCP/AACVPR evidencebased guidelines. Chest 1997;112:1363-96. 